\title{
Nanoscale Microstructure in a Titanium Aluminide Alloy
}

\author{
S.S.A. Gerstl, D. Reinhard, and D.J. Larson \\ Imago Scientific Instruments Corp., 5500 Nobel Drive, Madison, WI 53719-1193
}

The optimization of mechanical properties in titanium aluminide (TiAl) alloys has resulted in increasingly complex microstructure, and thus local microchemistries more difficult to measure. As with Ni-based superalloys, the properties of TiAl alloys have been pushed to new limits, due, in part, to nucleation of ultrafine lamellae (on the order of a few nanometers in thickness) during thermal treatments [1]. Observation and compositional measurement of these ultrafine lamella have become feasible using atom probe tomography (APT) [2,3].

The growth or dissolution (depending on the composition and thermal history) of such a nanolamella was resolved in three dimensions via laser mode APT (Fig. 1). The microstructural evolution of the lamella across the growth/dissolution 'front' was analyzed through $25 \mathrm{~nm}$ thick cross-sections with isoconcentration surfaces at 23\% Ti denoting the boundaries of the lamella (Fig. 2).

Historically, analysis parameters in APT consisted principally of voltage pulse fraction and base temperature [4]. In laser-pulsed atom probes, a specimen is held under a DC field while a thermal (rather than voltage) pulse is applied to the specimen apex. The heat transferred to the specimen briefly reduces the evaporation field necessary to allow atoms on the apex of the specimen to evaporate. The transfer of heat depends on complex factors as discussed in [5], but can be estimated based on the observed charge-state ratios of the constituent atoms because different temperatures of evaporation favor different charge states [6-8]. For this analysis the specimen was run at base temperatures of 60,120 and $180 \mathrm{~K}$ in the traditional voltage-pulsed mode at $15 \%$ pulse fraction, and then run in laser-pulsed mode at a base temperature of $20 \mathrm{~K}$ and laser energies of $0.4,0.5$ and $0.7 \mathrm{~nJ}$. The $\mathrm{Al}^{++} / \mathrm{Al}^{+}$charge-state ratio was determined from each data set (Fig. 3). A trendline was fit to each set of data and the temperature and laser energy axes were adjusted to make the trendlines overlap which confirms a linear relationship between laser energy and average evaporation temperature as Kellogg had suggested previously [9]. The charge-state ratios observed in this analysis suggest that the average evaporation temperature ranges from $200-400 \mathrm{~K}$. Lower laser powers are expected to reduce these numbers slightly in order to ensure a lower evaporation temperature regime.

[1] F. Appel and R. Wagner, Mat. Sci. Eng. R22, (1998) 187.

[2] D. J. Larson and M. K. Miller, Mat. Char. 44/1-2 (2000) 159.

[3] S.S.A.Gerstl et al. Adv. Mat.\&Proc.162 No.10 (2004) 31.

[4] M.K. Miller et al., Atom Probe Field Ion Microscopy, Oxford University Press, Oxford, 1996.

[5] J. H. Bunton et al., Microsc. Microanal. 13 (2007) 418.

[6] D.R. Kingham, Surf. Sci., 116 (1982) 273.

[7] M.K. Miller et al., Microsc. Microanal. 13(2) (2007) 1610.

[8] E. Marquis and B. Gault, J. Appl. Phys. 104 (2008) 084914.

[9] G.L.Kellogg, J. Appl. Phys. 52, 5320 (1981).

[10] Dr. Susan Draper at NASA Glen Research Center is thanked for provision of the material in this study. 


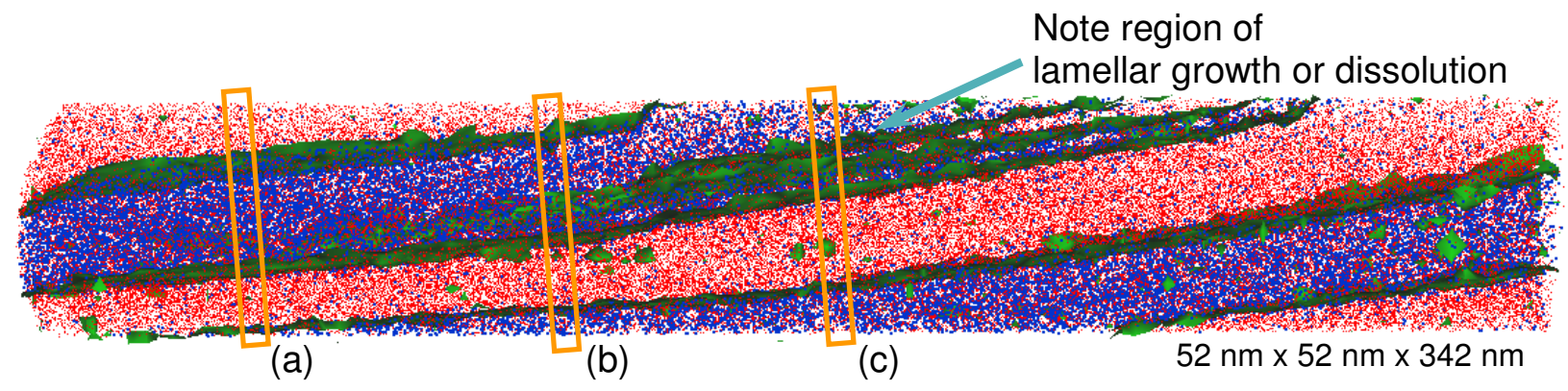

Fig. 1. Atom map with an isoconcentration surface at 23.0-at.\% $\mathrm{Al}$ delineating the two intermetallic phases: $\gamma$-TiAl and $\alpha_{2}-\mathrm{Ti}_{3} \mathrm{Al}$. Three 25 -nm slices refer to Figs. 2a-c.

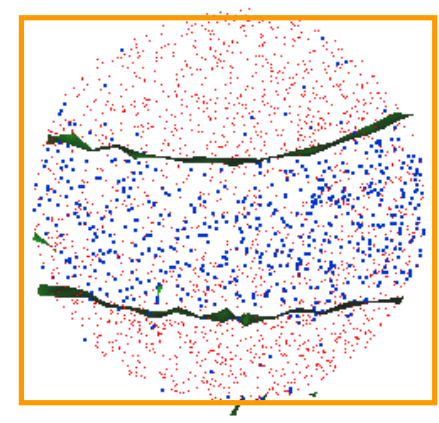

(a)

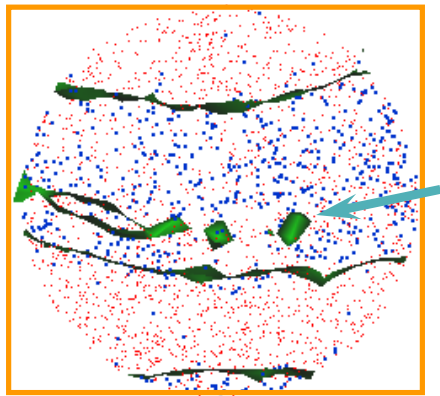

(b)

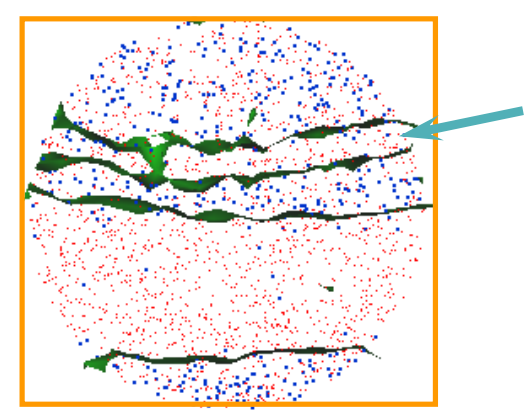

(c)

Fig. 2. (a-c) 25-nm slices through the three-dimensional reconstruction in Fig. 1 showing the growth or dissolution of an $\alpha_{2}$ lamella in the microstructure.

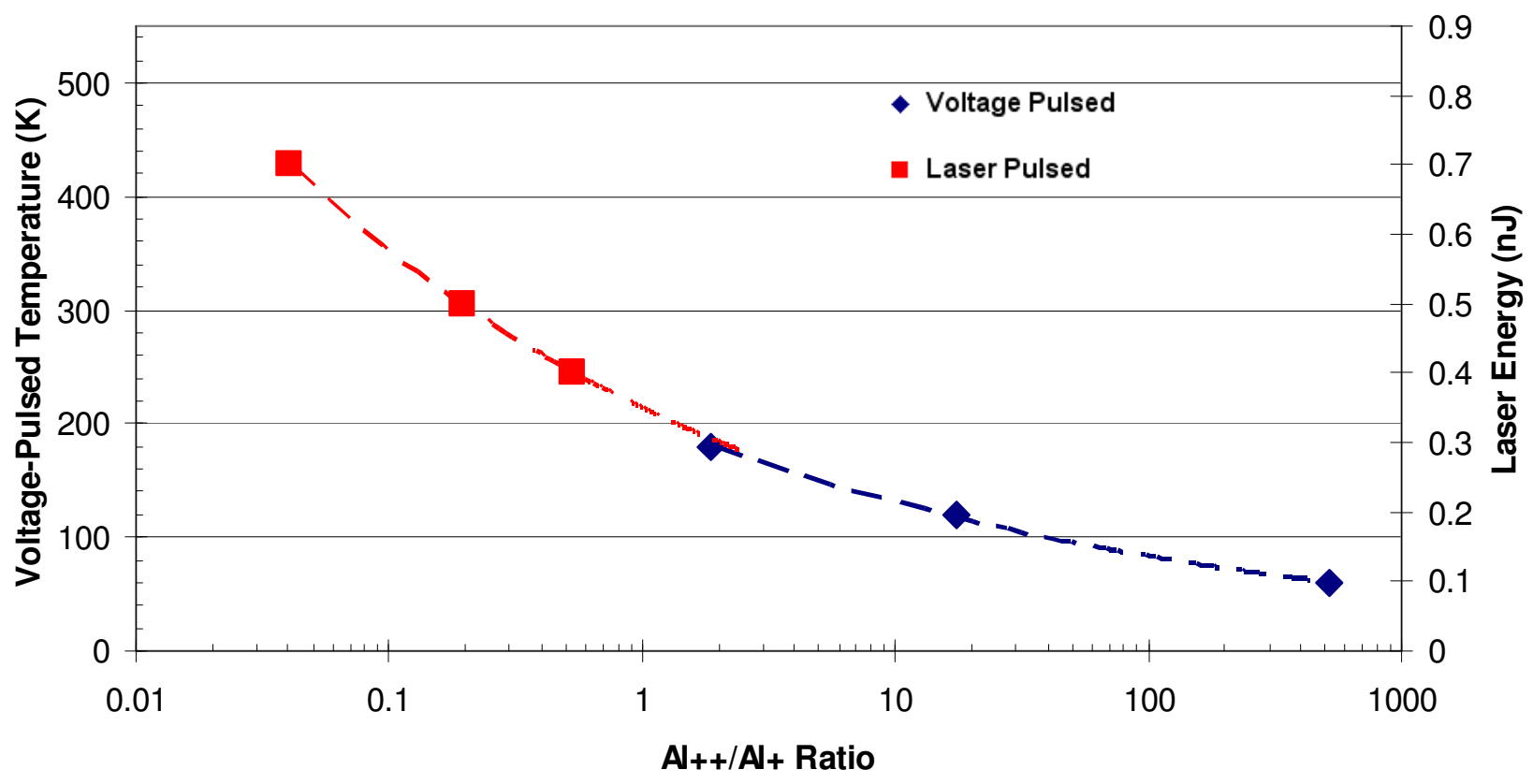

Fig. 3. Charge states of $\mathrm{Al}^{2+} / \mathrm{Al}^{+}$vs base temperature (voltage-pulsed, left axis) and $\mathrm{Al}^{2+} / \mathrm{Al}^{+}$vs laser energy (laser-pulsed, right axis). 